\title{
Development of a chemical model to predict the doses of calcium sulfate and tartaric acid to acidify musts in Sherry area
}

\author{
J. Gómez ${ }^{1, a}$, J.M. Palacios-Santander ${ }^{2}$, C. Lasanta ${ }^{1}$, L.M. Cubillana-Aguilera ${ }^{2}$, R. Arnedo ${ }^{3}$, J.A. Casas ${ }^{3}$, \\ B. Amilibia ${ }^{4}$, and I. LLoret ${ }^{5}$ \\ ${ }^{1}$ Department of Chemical Engineering and Food Technology, Faculty of Sciences, University of Cadiz, 11510 Puerto Real, Spain \\ ${ }^{2}$ Department of Analytical Chemistry, Faculty of Sciences, University of Cadiz, 11510 Puerto Real, Spain \\ ${ }^{3}$ Bodegas González-Byass, c/Manuel Maria González, 12, 11403 Jerez, Spain \\ ${ }^{4}$ Grupo Estévez, S.A. Carretera Nacional IV. Km 640, 11408 Jerez, Spain \\ ${ }^{5}$ Certification and Control Authority, Regulation Council of the Denomination of Origin Jerez-Xérèz-Sherry, Avda. Alcalde Álvaro \\ Domecq, 2, 11402 Jerez, Spain
}

\begin{abstract}
Calcium sulfate is normally used as a complementary acidifier combined with tartaric acid. The doses corresponding to each one depend on the desired reduction of $\mathrm{pH}$ and on the composition of musts. However, considering that there are several interrelated chemical equilibria implied (tartaric acid dissociation, calcium tartrate and potassium bitartrate precipitation, etc.), it is not easy to predict the effect on $\mathrm{pH}$ of a mixed tartaric acid and calcium sulfate addition and to determine the necessary doses to reach the final $\mathrm{pH}$ required by the winemaker. In a model previously developed by the authors, the prediction of $\mathrm{pH}$ after an acidification was properly achieved. On the contrary, in the same model the prediction of the necessary doses of acidifiers to achieve a desired $\mathrm{pH}$ have higher errors due to some parameters, as $\mathrm{pH}$ and $\mathrm{pK}$, are found as exponential functions in the equations. This work develops and verify the necessary corrections to the models so that appropriate predictions of the doses are obtained. With these corrections, prediction errors of less than $5 \%$ were obtained for all doses of acidifiers, confirming the good comprehension of the chemical equilibria involved in this practice.
\end{abstract}

\section{Introduction}

The use of calcium sulfate $\left(\mathrm{CaSO}_{4} \cdot 2 \mathrm{H}_{2} \mathrm{O}\right)$ is authorized in the European Union as a complementary acidifier in liquor wines from Spain provided that the residual sulfate content in the wine does not exceed $2.5 \mathrm{~g} / \mathrm{L}$ expressed as potassium sulfate [1]. Calcium sulfate is also authorized in the United States for the production of wines aged under yeast veil although residual sulfate cannot exceed $2 \mathrm{~g} / \mathrm{L}$ [2]. The OIV is also currently considering the approval of calcium sulfate for liquor wines [3]. Gómez et al. [4] recommended a combined acidification with $2 \mathrm{~g} / \mathrm{L}$ of calcium sulfate and sufficient tartaric acid to achieve a $\mathrm{pH}$ of 3.25 . In this way, the necessary dose of tartaric acid does not exceed $1.5 \mathrm{~g} / \mathrm{L}$, the maximum authorized level in UE, and the final concentration of sulfates is lower than $2.5 \mathrm{~g} / \mathrm{L}$. To predict the final $\mathrm{pH}$ after an addition of tartaric acid or/and calcium sulfate Moreno and Peinado [5] proposed a simple and easy to apply model. In this model the acidity of wine is considered to be due to a monoprotic acid. This model has been developed and verified in [6,7], obtaining errors in $\mathrm{pH}$ predictions lower than 5\%. However for winemakers is more interesting to predict the necessary acidifier dose to reach the required $\mathrm{pH}$. In this way the OIV prescribes in [3] that "It is advisable to make previous tests in the laboratory to calculate the doses of calcium sulfate and tartaric acid needed to reach the required $\mathrm{pH}^{\prime}$.

\footnotetext{
a e-mail: juangomez.benitez@uca.es
}

To predict the dose of acidifiers it is necessary to change the equations proposed in [6] in this way:

For tartaric acid:

$$
X=\frac{A A i-T A i \times 10^{p H f-p K i}}{1+10^{p H f-p K i}}
$$

where $X$ is the dose in meq/L, $K_{i}$ is the acidity constant, $A A$ is the ash alkalinity and $T A$ is the total acidity. The subscripts " $i$ " and "f" denote initial and final states respectively. In this model it is considered that the medium is saturated in tartrate and, as a consequence, the addition of tartaric acid as an acidifying agent will introduce the common ion $\mathrm{HT}^{-}$(bitartrate) and this will precipitate naturally or during cold stabilization as potassium bitartrate. In this way, one can consider that all of the $\mathrm{HT}^{-}$added will precipitate as potassium bitartrate and this does not contribute to the titratable acidity.

\section{For calcium sulfate}

$$
Y=\frac{A A i-T A i \times 10^{p H f-p K i}}{1-10^{p H f-p K i}}
$$

and for combined acidification, the final equation would be a combination of both.

Nevertheless, in these equations $\mathrm{pH}$ and $\mathrm{pK}$ appears as exponential functions what increase the effect of the errors. In consequence, an error of 0.1 units of $\mathrm{pH}$ produces an error of $25 \%$ in the predicted dose. 


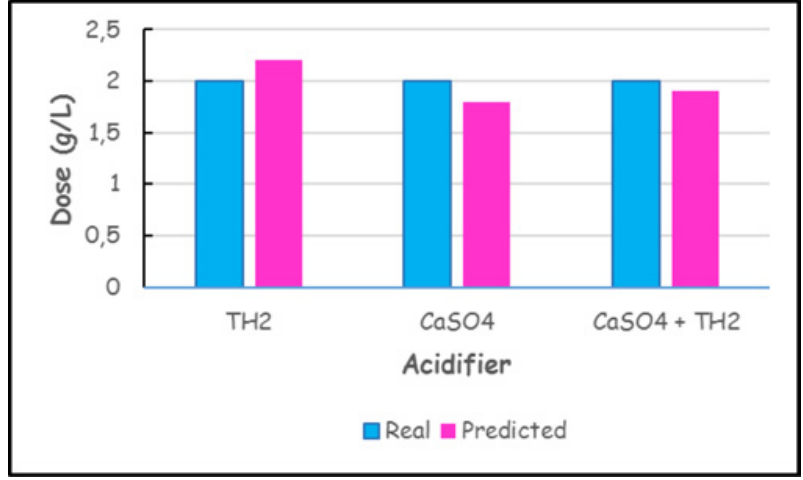

Figure 1. Prediction of the doses of acidifiers.

Experiences carried out in the harvest of 2016 working with the best laboratory conditions (Fig. 1) and described in [7] showed that the errors in the prediction of the acidification doses cannot be reduced below $10 \%$ using the model described in [6].

This fact shows that the simplified model used must be corrected to obtain better prediction results.

The aim of this work consists of designing and verifying some corrections in the model previously used in order to minimize the errors in the prediction of the doses of acidifiers used in the winemaking of Sherry musts.

\section{Materials and methods}

\subsection{Corrections to the model}

\section{For Tartaric acid:}

Instead of supposing that all added tartaric acid precipates as potassium bitartrate, it is considered that a fraction $\mathbf{f}$ of the addition does not precipitate because there is not enough time to reach the equilibrium in acidified musts before fermentation. In this case, the dose X of tartaric acid would be obtained by a new equation to be called (3).

\section{For Calcium sulfate}

The initial model does not consider the increase of ash alkalinity due to calcium sulfate. In consequence, in this correction it is considered that fraction $\mathbf{g}$ of added sulfate remains in solution and increases ash alkalinity. The dose $\mathrm{Y}$ of calcium sulfate would be obtained by e new equation to be called (4).

\section{For combined acidification}

A combination of the equations (3) and (4) is used in combined acidification. The dose $\mathrm{X}$ of tartaric acid would be obtained by the Eq. (5) where logically participates the dose $\mathrm{Y}$ of calcium sulfate.

\subsection{Acidification tests}

The Sherry must used in the acidification tests was obtained from Palomino fino grapes harvested at optimum maturation state and pressed in a pneumatic press at a pressure lower than $1 \mathrm{Kg} / \mathrm{cm}^{2}$. Next, $2 \mathrm{~g} / \mathrm{L}$ of $\mathrm{SO}_{2}$ was added to the obtained must to allow its preservation until
Table 1. Results of the acidification tests.

\begin{tabular}{|c|c|c|c|c|}
\hline $\begin{array}{c}\text { Tartaric } \\
\text { acid dose } \\
(\mathrm{g} / \mathrm{L})\end{array}$ & $\begin{array}{c}\text { Calcium } \\
\text { sulfate } \\
\text { dose } \\
(\mathrm{g}(\mathrm{L})\end{array}$ & $\begin{array}{c}\text { Titratable } \\
\mathbf{p H} \\
(\mathrm{meq} / \mathrm{L})\end{array}$ & $\begin{array}{c}\text { Buffering } \\
\text { acidity } \\
(\mathrm{meq} / \mathrm{L} \cdot \mathrm{u} \cdot \mathrm{pH})\end{array}$ & power \\
\hline 0 & 0 & 3.71 & 50.38 & 38.70 \\
\hline 1.0 & 0 & 3.52 & 64.78 & 43.00 \\
\hline 0 & 1.0 & 3.66 & 49.14 & 36.19 \\
\hline 1.0 & 1.0 & 3.44 & 65.52 & 41.03 \\
\hline 1.5 & 0 & 3.48 & 69.74 & 49.02 \\
\hline 0 & 1.5 & 3.60 & 50.88 & 33.68 \\
\hline 1.5 & 1.5 & 3.36 & 70.74 & 46.80 \\
\hline 2.0 & 0 & 3.41 & 79.93 & 55.05 \\
\hline 0 & 2.0 & 3.63 & 50.66 & 31.17 \\
\hline 2.0 & 2.0 & 3.26 & 79.68 & 53.78 \\
\hline
\end{tabular}

the acidification tests were carried out and to avoid the beginning of alcoholic fermentation. These tests were conducted with $100 \mathrm{~mL}$ of must in $250 \mathrm{~mL}$ Erlenmeyer flaks, placed on a magnetic stirrer and adding slowly the corresponding dose of acidifier weighed with a precision of $0.1 \mathrm{mg}$. The flasks were covered with plastic film to avoid $\mathrm{SO}_{2}$ evaporation. For the first 2 minutes the stirring speed was high to dissolve the acidifiers and later it was maintained until 30 minutes at the lowest possible speed (c.a. 60 r.p.m.) to keep the solids suspended. The doses of tartaric acid and calcium sulfate used were 1.0, 1.5 and $2.0 \mathrm{~g} / \mathrm{L}$ alone and combined. The samples were centrifuged before analysis and $\mathrm{pH}$, titratable acidity and buffering power were determined in all samples before and after acidification. All tests and analysis were carried out in duplicate indicating the results as the average values.

\subsection{Analytical methods}

$\mathrm{pH}$, titratable acidity and buffering power were determined as in [6].

\subsection{Determination of $f$ and $g$ factors}

Iterative calculations using Excel 2016 were carried out to know the values of $f$ and $g$ factors that minimized the errors in the predicted doses of acidifiers obtained by using the Eqs. (3), (4) and (5) in relation to the real doses. In these calculations, $f$ and $g$ factors were gradually changed until obtaining the minimal errors. In the combined acidification, the dose of tartaric acid was fixed and we tried to predict the dose of calcium sulfate. In consequence, couples of $f$ and $g$ factors values minimizing the error in calcium sulfate dose were searched for.

\section{Results and discussion}

\subsection{Acidification tests}

The results of the acidification tests carried out in the laboratory are shown in Table 1 . In general terms, these results are according with those obtained in previous research works [6].

\subsection{Determination of $f$ and $g$ factors}

The evolutions of errors with the values of $f$ and $g$ factors for an acidification of $2 \mathrm{~g} / \mathrm{L}$ are shown in Figs. 1 and 2 


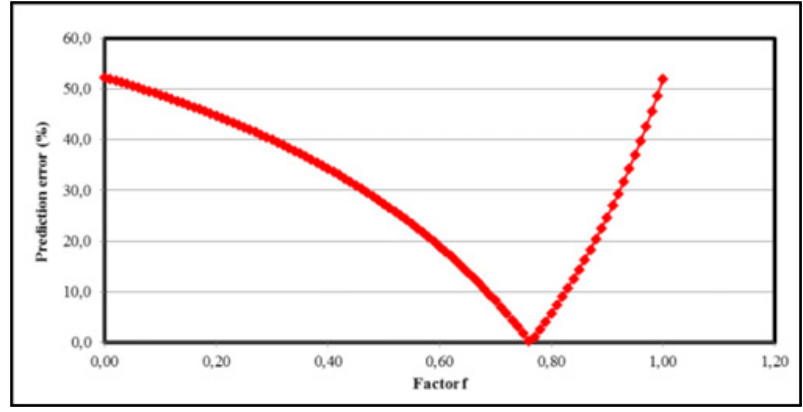

Figure 2. Variation of the prediction error with $f$ factor for acidification with $2 \mathrm{~g} / \mathrm{L}$ of tartaric acid.

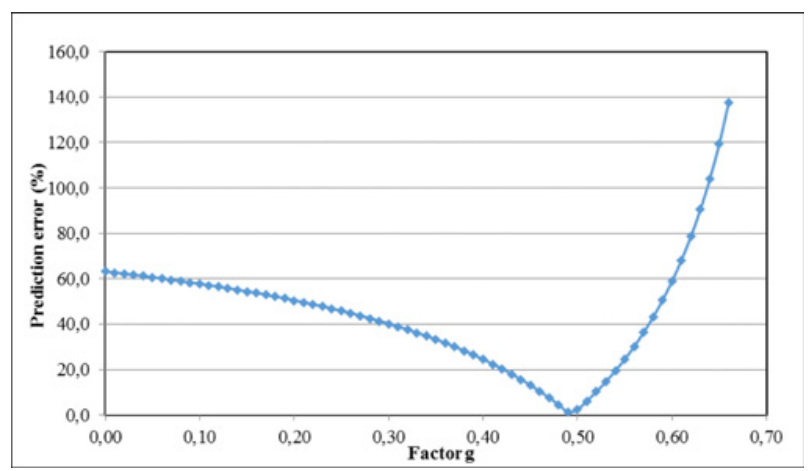

Figure 3. Variation of prediction error with $g$ factor for acidification with $2 \mathrm{~g} / \mathrm{L}$ of calcium sulfate.

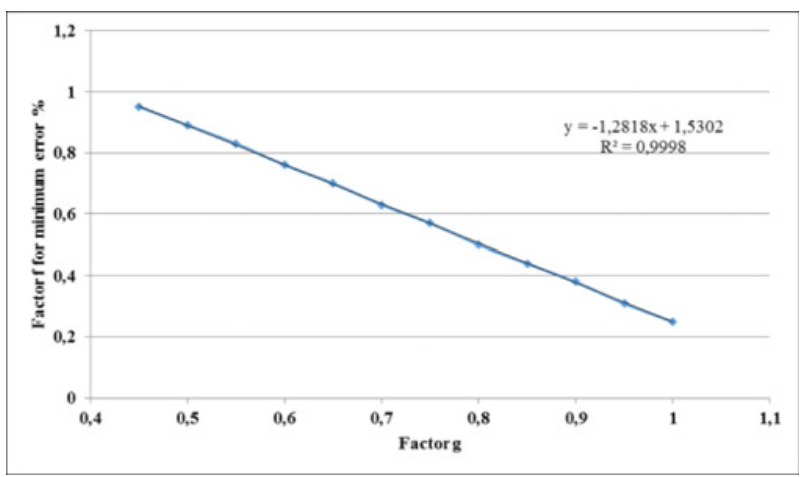

Figure 4. Values of $f$ and $g$ factors in combined acidification with $2 \mathrm{~g} / \mathrm{L}$ of tartaric acid and calcium sulfate.

Table 2. $f$ and $g$ factors for different doses of acidifiers.

\begin{tabular}{|c|c|c|}
\hline $\begin{array}{c}\text { Acidifier dose } \\
(\mathrm{g} / \mathrm{L})\end{array}$ & $\begin{array}{c}f \text { factor } \\
\text { (Tartaric acid) }\end{array}$ & $\begin{array}{c}g \text { factor } \\
(\text { Calcium Sulfate })\end{array}$ \\
\hline 2 & 0.76 & 0.49 \\
\hline 1.5 & 0.80 & 0.53 \\
\hline 1 & 0.80 & 0.55 \\
\hline
\end{tabular}

As it can be seen, both evolution plots show similar shapes, first decreasing the error with the factors until reaching a minimum value, corresponding to certain factor value, and then sharply increasing the prediction errors with the factor values.

Figure 4 shows the values of $f$ and $g$ factors that minimize the errors in combined acidification. As it can be seen, these factors are very well correlated with a correlation factor of 0.9998 .

The procedure for calculating the $f$ and $g$ values for other acidifier doses was the same and the values obtained are shown in Table 2. As it can be seen, the factors are approximately constants for all considered doses. This fact confirms that the corrections of the model considered are valid regardless of the doses of acidifiers used.

\section{Conclusions}

The proposed corrections to the chemical model for the acidification equilibria in the Sherry winemaking are able to predict properly the necessary doses to reach the required $\mathrm{pH}$ values with very low errors. Hence, the chemical model developed can be a valuable tool for winemakers that can be easily implemented as a spreadsheet in a computer to be used in the laboratory during the harvest.

\section{References}

[1] European Union, Commission Regulation (EC) $\mathrm{N}^{\circ} 606 / 2009$ laying down the categories of grapevine products, oenological practices and the applicable restrictions, O.J.E.U. L153(606/2009), pp. 1-59 (2009)

[2] e-CFR.Electronic Code of Federal Regulations, "Title 27: Alcohol, Tobacco and Firearms PART 24 WINE Subpart L Storage, Treatment and Finishing of Wine $\$ 24.246$ Materials authorized for the treatment of wine and juice. Available on line at http://www.ecfr.gov/cgi-bin/text-idx?SID= 5a39ba6737562446636," 2010

[3] OIV, "Resolution OENO-TECHNO 15-583 Step 7. Treatment of musts with calcium sulphate for liqueur wines." 2017

[4] G. J, G. MM, and D. J, "Study of the acidification of Sherry musts with gypsum and tartaric acid," Am. J. Enol. Vitic. 44(4), pp. 1-5 (1993)

[5] J. Moreno Vigara and R. A. Peinado Amores, Oenological Chemistry, 1st ed. San Diego CA. (USA): Academic Press, 2010.

[6] J. Gómez, C. Lasanta, J. M. Palacios-Santander, and L. M. Cubillana-Aguilera, "Chemical modeling for $\mathrm{pH}$ prediction of acidified musts with gypsum and tartaric acid in warm regions," Food Chem. 168, pp. 218-224 (2015)

[7] J. Gomez et al., "Comprehensive chemical study of the acidification of musts in Sherry area with calcium sulphate and tartaric acid.," in 39th World Congress of Vine and Wine (2016) 